\title{
Diagnostic and Management of Patient with Adrenocortical Carcinoma
}

\author{
Mamluatul Karimah', Hermina Novida ${ }^{2 *}$ \\ ${ }^{1}$ Department of Internal Medicine, Faculty of Medicine, Universitas Airlangga - Dr. Soetomo General Hospital Surabaya, \\ Indonesia. \\ ${ }^{2}$ Division of Endocrinology and Metabolic, Department of Internal Medicine, Faculty of Medicine, Universitas Airlangga - \\ Dr. Soetomo General Hospital Surabaya, Indonesia.
}

\begin{abstract}
A B S T R A C T
Endogenous Cushing's syndrome is a rare case, and about $8 \%$ is due to adrenocortical carcinoma. We report a case of a 31-year-old woman with complaints of weight gain, round face, brown lines, menstrual disorders, and limb weakness. On physical examination, there were hypertension, moon face, central obesity, and stroke. On laboratory examination, there were morning serum cortisol levels were 46.87 and ACTH levels 5. On CT scan. It was found that the right adrenal gland solid mass enhancement was $+/-6.6 \times 4.9 \times 7.3 \mathrm{~cm}$, with suspicion of adrenal carcinoma. On histopathological examination, adrenocortical carcinoma was obtained, and capsule invasion was obtained. Patients underwent surgery to remove the tumor, radiotherapy and chemotherapy. The patient was alive and had no residual mass at the surgical bed.
\end{abstract}

Keywords: Adrenal Carcinoma, Cushing's Syndrome, Adrenal mass

Correspondence: Hermina Novida

E-mail: hermina-n@fk.unair.ac.id

Article history: • Received 21 December $2020 \bullet$ Received in revised form 28 January $2021 \bullet$ Accepted 29 January 2021

\section{INTRODUCTION}

Cushing's syndrome is a syndrome that results from chronic exposure to excess glucocorticoid hormones in the blood. Both endogenous and exogenous factors can cause Cushing's syndrome. Exogenous factors that cause Cushing's syndrome are exposure to glucocorticoids from outside the body, while endogenous factors that cause Cushing's syndrome can be divided into ACTHdependent and ACTH-independent. ACTH-independent occurs in about $20 \%$ of people with endogenous Cushing's syndrome and about $8 \%$ due to adrenocortical carcinoma. Adrenocortical carcinoma is found in many young patients and children, so it needs proper diagnosis and aggressive management. It has been found that further management of adrenocortical carcinoma but with limited efficacy and significant side effects can reduce patients' quality of life (Rajput, 2013; Terzolo et al, 2013).

\section{CASE REPORT}

Mrs. R, 31 years old, female, Batak ethnicity, Catholic, married, residing in Sidoarjo, East Java, BPJS financing. Come to the Endocrine Clinic, dr. Soetomo on May 1, 2017, with a chief complaint of brownish lines on the skin.

From the history, it was found that brown streaks appeared on the body's skin six months before the patient came to the hospital. Initially, brown streaks appeared on both legs, then appeared on the patient's stomach. The patient also complained of gaining weight with relatively smaller legs for six months before the patient came to the hospital. The patient's face also felt increasingly swollen. The patient complained that the skin on the body was getting whiter, thinner, and drier. The patient also felt tired quickly, and the body was often weak. The patient also complained that acne often appeared for seven months before coming to the hospital so that the patient took acne medication from the pharmacy, and the patient forgot the name of the medicine. The patient denied the frequent complaint of swelling in both legs. She also denied the history of frequent fever, shortness of breath. Complaints of frequent hair loss and easy removal were experienced for six months before coming to the hospital. The patient has not had her period for five months. There were no complaints on urination and defecation. The patient had no history of diabetes mellitus, hypertension, asthma, heart disease, tumors, and had never been hospitalized before. The patient's family did not have the same history of pain as the patient.

The patient had a history of using monthly injectable contraceptives since 2014 and stopped one year ago. The patient denied the history of taking any other drugs.. The patient married in 2013 and has one child who was currently 3 years old. The patient was currently working as a kindergarten teacher. The patient did not smoke.

On physical examination, the general condition is good, with GCS 456, blood pressure 139/89, pulse 92x/minute, breathing $16 \mathrm{x}$ per minute, and axillary temperature $36.7^{\circ} \mathrm{C}$. The examination of the head and neck showed moon face, hyperpigmentation of the lip mucosa, no anemia, jaundice, 
cyanosis, or dyspnoea. The chest examination obtained S1, S2, without additional sound murmurs or gallops. Lung examination showed no additional breath sounds and vesicular breath sounds in both lung fields. On examination of the abdomen, there was central obesity with striae, and no shifting dullness was found. Examination of the limbs revealed striae in both thighs to knees, warm acral, and CRT $<2$ seconds. There were no enlarged lymph nodes in the armpit and later.

From laboratory tests, it was obtained GDP $110 \mathrm{mg} /$ dl, GD2JPP 253mg/dl, SGOT 18U/L, SGPT 42U/L, BUN $9 \mathrm{mg} / \mathrm{dL}$, serum creatinine $0.7 \mathrm{mg} / \mathrm{dL}$, uric acid $4.3 \mathrm{mg} /$ $\mathrm{dL}$, potassium $3.7 \mathrm{mmol} / \mathrm{l}$, sodium $148 \mathrm{mmol} / \mathrm{L}$, chloride $95 \mathrm{mmol} / 1$, total cholesterol $315 \mathrm{mg} / \mathrm{dL}$, triglycerides $147 \mathrm{mg} / \mathrm{dL}$, HDL $85 \mathrm{mg} / \mathrm{dL}$, LDL $179 \mathrm{mg} / \mathrm{dL}$, Cortisol morning serum 46.87ug/dL (4, 3-22.40), ACTH 5 pg/ $\mathrm{mL}(<46)$. On plain chest radiograph examination, the cast and Pulmo showed no abnormalities. On abdominal ultrasound examination, multiple nodules were found in the liver. From the CT scan of the abdomen with contrast, the results were: There was a solid mass (34HU), firm borders, lobulated edges, the size of $+/-6,6 \times 4,9,7,3 \mathrm{~cm}$ in the right adrenal gland with contrast visible enhancement venous phase (85HU), delay (63HU) (relative percentage washout) appeared to be a small part of penetrating the capsule. There was no visible expansion to the kidneys or liver. The liver, pancreas, spleen of Ren D et S, uterus, and adnexa were not seen. There was no visible extraluminal free fluid density in the abdominal cavity and pleural cavity right and left with a conclusion. Enhancing solid mass of right adrenal gland with size $+/-6,6 \times 4,9 \times 7,3 \mathrm{~cm}$ with relative percentage when washing out, a small part penetrated the capsule, suspicious. Adrenal carcinoma and bilateral renal caliectasis could be caused due to a full bladder. The patient was diagnosed with an adrenal tumor suspiciously malignant with Cushing's syndrome. The patient was referred to the urology department, and surgery was planned. The patient underwent laparotomy adrenalectomy by a urology colleague. The patient was subjected to postoperative histopathological examination and obtained the adrenal cortical carcinoma results, and obtained invasion from the capsule. The patient was diagnosed with adrenocortical carcinoma stage 4 T3 No M1. Patients were outpatient and radiotherapy, and chemotherapy were planned.

After 1 month of adrenalectomy, the patient's complaint of swelling on the face and body decreased, the patient's weight also felt to decrease. In laboratory examination, morning serum cortisol results were 11.63, GDP 112. The patient was consulted with the radiodiagnostic department for radiotherapy considerations.

In the third month after tumor resection, the patient had no complaints. The swelling in the patient's body was felt to have disappeared, the patient's weight was also back to normal. The patient was planned to receive 25 times radiotherapy in the Radiodiagnostic Department of Dr. Soetomo Hospital, Surabaya.

In the fifth month after tumor resection, after radiotherapy was performed on the patient, the patient was given chemotherapy with a regimen of etoposide, doxorubicin, and cisplatin for 8 cycles. After chemotherapy, the patient was evaluated with an abdominal CT scan, and the results showed no visible mass in the abdominal cavity, no residual or residual mass on the surgical bed.

\section{DISCUSSION}

Cushing's syndrome is a syndrome that results from chronic exposure to excess glucocorticoid hormones in the blood. Both endogenous and exogenous factors can cause Cushing's syndrome. Cushing's syndrome due to endogenous factors is very rare and is divided into ACTH-dependent and ACTH-independent. ACTHdependent Cushing's syndrome can result from excess ACTH secretion from the pituitary gland or ectopic ACTH production, which is usually the result of a neuroendocrine tumor. The ACTH hormone produced by pituitary tumors and those produced by non-pituitary tumors can stimulate the hormone cortisol production from the adrenal glands. ACTH-independent Cushing's syndrome is caused by excess production of cortisol by the adrenal glands, usually due to benign adrenal tumors or adrenocortical adenomas, while those caused by adrenocortical carcinoma are rare. This excess cortisol production will inhibit $\mathrm{CRH}$ and ACTH's secretion from the pituitary gland (Nieman \& Swearingen, 2013; Raff et al, 2014). The mechanism of Cushing's syndrome can be seen in the image below.

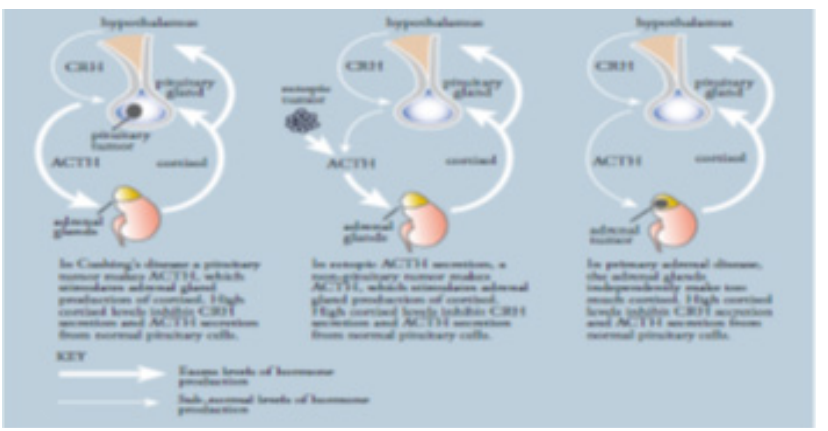

Figure 1. mechanism of Cushing's syndrome (Nieman \& Swearingen, 2013)

The diagnostic criteria for Cushing's syndrome are obtained from history, physical examination, and laboratory examination. In Cushing's syndrome, high levels of glucocorticoids in the blood for a long time can cause abnormalities in the metabolic system such as central obesity, weight gain, hyperglycemia, dyslipidemia, hypokalemia, hormonal disorders (menstrual disorders or amenorrhea, delayed puberty), disorders of the musculoskeletal system. (muscle weakness and osteoporosis), disorders of the cardiovascular system (hypertension, venous thromboembolism) and skin manifestations (striae, facial plethora), and neuropsychological manifestations (insomnia, depression, and irritability). Radiological examination in patients with Cushing's syndrome is useful to determine the tumor's location as a cause. In laboratory tests, several tests are needed to help make a diagnosis of Cushing's syndrome. Three laboratory tests required to diagnose Cushing's syndrome are a 24-hour urine cortisol test, morning serum cortisol, and a low-dose dexamethasone suppression test. To see the cause of Cushing's syndrome, several tests can be performed, namely, the CRH stimulation 
test, High dose dexamethasone suppression test (HDDST), and radiological examinations to see first hand the picture of the endocrine glands (Nieman et al, 2005). The steps to find out the cause of Cushing's syndrome can be seen in the image below.

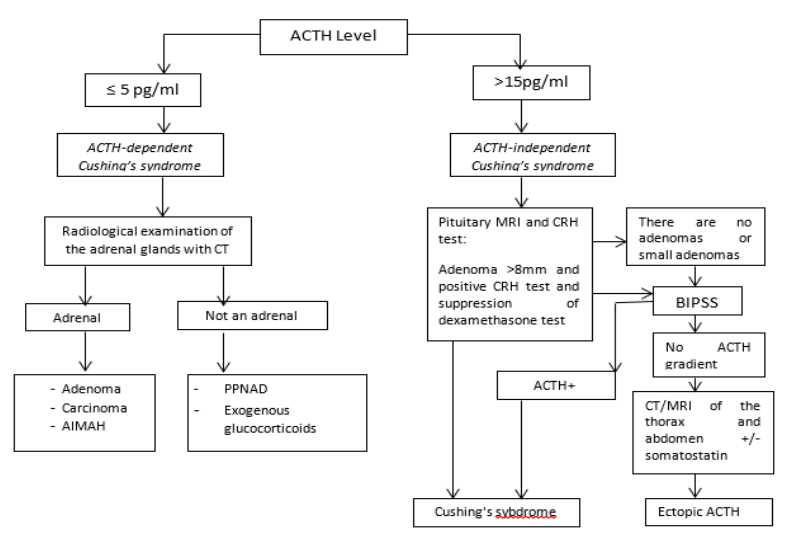

Figure 2. Diagnosis and etiology of Cushing's syndrome (Proce \& Grossman, 2007).

To determine the cause of Cushing's syndrome, the first step that must be done is to check the ACTH levels in the blood. If the ACTH level in the blood is below 15pg/ $\mathrm{ml}$, these results indicate ACTH-independent Cushing's syndrome, and in the next step, a CT or MRI scan of the adrenal glands should be performed. If the ACTH level is above $15 \mathrm{pg} / \mathrm{ml}$ then these results indicate an ACTHdependent Cushing's syndrome, and in the next step, a CT scan or MRI examination of the pituitary gland is performed, a CRH test, and a high-dose dexamethasone suppression test (HDDST). If the ACTH plasma level is above 5 to 15 then careful interpretation and further examination with the CRH test is needed (Price \& Grossman, 2007).

The patient complained of weight gain, rounded face, brownish lines on the skin, menstrual disorders, and limb weakness. On physical examination, hypertension, moon face, central obesity, and stroke were found. On laboratory tests, GD2JPP 253, total cholesterol 315, LDL 179, morning serum cortisol levels 46.87, and ACTH 5 levels. On CT scan, it was obtained solid mass enhancement of right adrenal gland with a size of $+/-6.6 \times 4.9 \times 7.3 \mathrm{~cm}$. with a relative percentage when washing out, a small portion penetrated the capsule, suspecting adrenal carcinoma.

Adrenocortical carcinoma is a rare malignancy with an incidence of 0.7 - 2 cases per million population per year. Most patients show symptoms of excess steroid hormones in the body or a mass in the abdomen, but about $15 \%$ of patients with adrenocortical carcinoma are found incidentally. Adrenocortical carcinoma can occur in various age groups, and women are more often affected than men, which is about $50-60 \%$. The diagnosis of adrenocortical carcinoma requires examination in the field of endocrine, radiology, and pathology. Most patients show symptoms of excess steroid hormones in the body (glucocorticoids, mineralocorticoids, and androgens) or the effects of an abdominal mass, and about $15 \%$ of patients with adrenocortical carcinoma are diagnosed incidentally first. The hormonal tests required for adrenocortical carcinoma diagnosis are basal cortisolACTH, dehydroepiandrosterone, testosterone, estradiol, and androstenedione. Radiological examination is needed to help make the diagnosis of adrenocortical carcinoma. In general, adrenocortical carcinoma is large and heterogeneous, with irregular margins. Hemorrhagic or calcified parts sometimes accompany it. Tumor size is needed to determine the type of tumor. Adrenocortical carcinoma generally has a size of $>11 \mathrm{~cm}$, while cortical adenoma generally has a size of $<5$ $\mathrm{cm}$. There is currently no single radiological examination that can be used to help establish adrenocortical carcinoma. An abdominal CT scan should be performed in patients with suspected adrenocortical carcinoma. If the abdominal CT scan shows a basal density $\leq 10 \mathrm{HU}$, it can be suspected that it is a benign lesion. If the basal density is $>10 \mathrm{HU}$ and the administration of contrast media in the washout phase helps differentiate benign lesions from adrenocortical carcinoma. If the washout phase is $>50 \%$, a benign lesion can be suspected. In addition to an abdominal CT scan, other radiological examinations are also important to show the process of the spread of the tumor. In addition to the radiological examination, an important examination to establish adrenocortical carcinoma diagnosis is histopathological examination. Histopathological examination is used to determine a definite diagnosis of adrenocortical carcinoma. Examination using the FNAB for the biopsy is rarely used because of the risk for needle track metastases, and it is difficult to distinguish whether the lesion is benign or malignant. All patients with adrenal tumors should rule out pheochromocytoma in preparation for surgery (Libe, 2015; Zini L I, 2011).

The patient showed symptoms of Cushing's syndrome and ACTH 11 levels. A solid mass (34HU), firm boundaries, lobulated edges, size $+/-6,6 \times 4,9,7,3 \mathrm{~cm}$ were found in the right adrenal gland with contrast administration on the CT scan. It appears that the contrast enhancement in the venous phase (85HU), delay (63HU) (relative percentage washout) appears to be a small part through the capsule. On histopathological examination, adrenocortical carcinoma and capsule invasion were obtained.

After someone is diagnosed with adrenocortical carcinoma, the next step is to determine the tumor stage. One of the classifications that use the metastatic nodule tumor (TNM) system to determine adrenocortical carcinoma patients' stage is the ENSAT system. In stage 1, there was a tumor with a size of $\leq 5 \mathrm{~cm}$, and in stage 2 , there was a tumor size of $>5 \mathrm{~cm}$, both without finding any spread to the surrounding tissue or far. In stage 3 , there is infiltration to the surrounding tissue, lymph nodes, or thrombus in the vena cava and/or renal veins, while in stage 4 there are distant metastases from the tumor. The prognosis of adrenocortical carcinoma is determined by tumor stage, resection status, and tumor grading. The survival rate of adrenocortical carcinoma is divided into 3 groups. First, localized tumors, namely tumors that do not invade the surrounding tissue, obtained a 5-year relative survival of $65 \%$. The second group is regional tumors, namely tumors that invade the surrounding tissue (stage 3 ), with a 5-years survival rate of $44 \%$, and the third group is tumors that have distant spread or metastasis, with 5 years survival rates of $7 \%$ (Libe, 2015). In this patient, on abdominal ultrasound examination, there were multiple nodules on the liver. The 
chest X-ray showed no abnormalities.

Treatment of adrenocortical carcinoma consists of surgery, radiation, and chemotherapy. Adrenocortical carcinoma management requires cooperation from various divisions, namely endocrinologists, surgeons, interventional radiologists, and oncologists. The management of patients with adrenocortical carcinoma can be seen in the picture below.

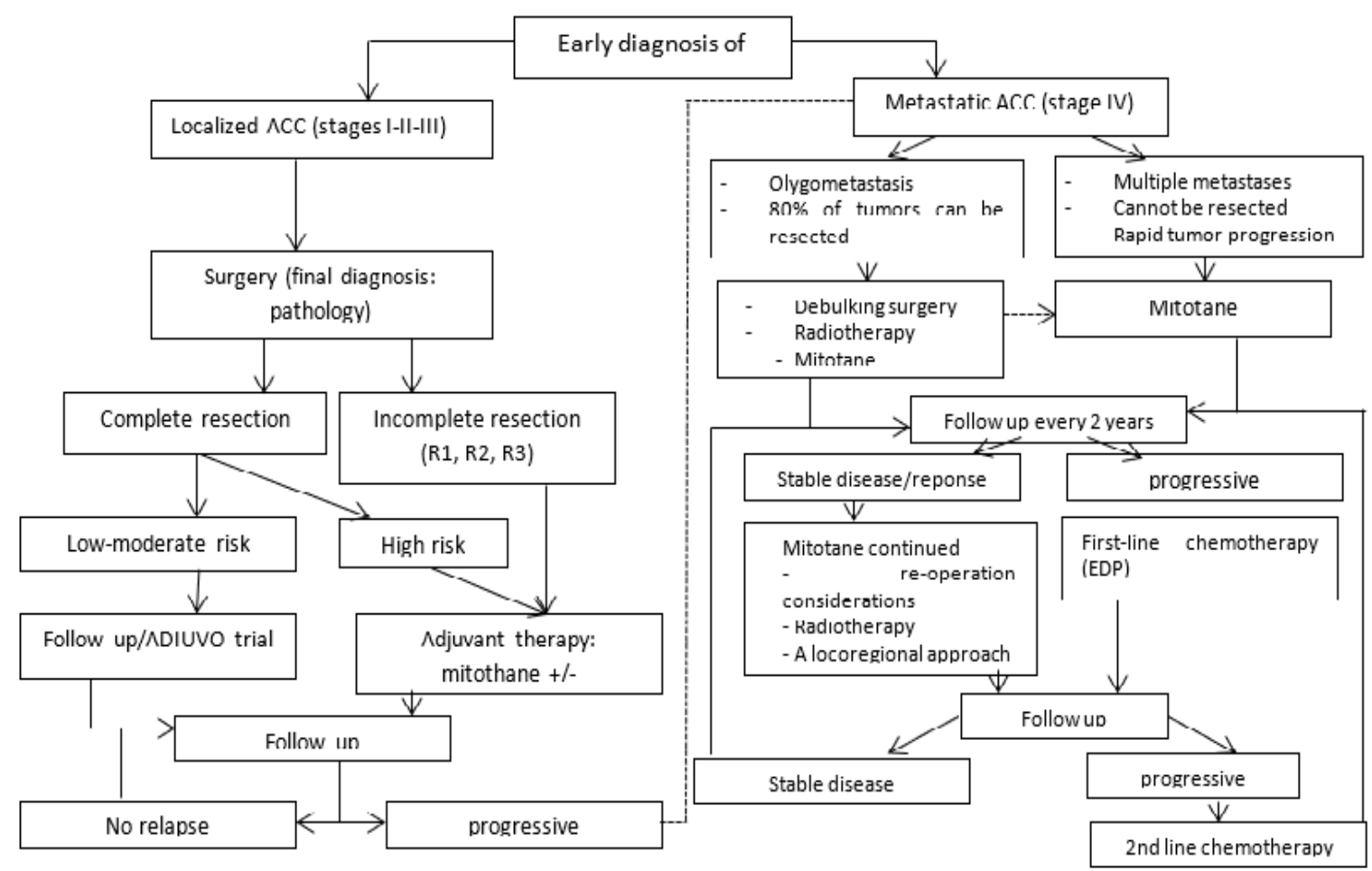

Figure 3. management of adrenocortical carcinoma (Libe, 2015)

In patients with adrenocortical carcinoma with localized tumors, surgery with total tumor removal is still the primary therapy. Adrenalectomy is also indicated in patients with stage I and II adrenocortical carcinomas, according to ENSAT. Besides, resection is indicated in ENSAT stage III if evidence of loco-regional invasion, including the extension to adjacent organs or the vena cava, is indicated. Another therapy for adrenocortical carcinoma is radiotherapy. Radiation therapy in patients with adrenocortical carcinoma aims to reduce the risk of local recurrence of the tumor. In stage 4, adrenocortical carcinoma with oligometastatic (metastasis in 1 organ) and $>80 \%$ of the tumor can still be resected, tumor resection can be done, radiotherapy and chemotherapy with mitotane are given, whereas, in patients with multiple metastases, mitotane chemotherapy can be given only. Then the patient is evaluated every 2 months, and if progressive results are obtained, then first-line chemotherapy with etoposide, doxorubicin, and cisplatin can be given. If there is stable disease or response, then chemotherapy with mitotane can be continued (Libe, 2015).

In this patient, surgical removal of the tumor was carried out, followed by 25 radiotherapies and chemotherapy with etoposide, doxorubicin, and cisplatin 8 times.

\section{CONCLUSION}

It has been reported that a female patient presents with complaints of weight gain, round face, brownish lines, menstrual disorders, and limb weakness. On physical examination, hypertension, moon face, central obesity, and stroke were found. On laboratory tests, GD2JPP 253, total cholesterol 315, LDL 179, morning serum cortisol levels 46.87, and ACTH 5 levels. On CT scan, it was obtained solid mass enhancement of right adrenal gland with a size of $+/-$ $6.6 \times 4.9 \times 7.3 \mathrm{~cm}$. with a relative percentage when washing out, a small portion penetrated the capsule, suspecting adrenal carcinoma. On histopathological examination, adrenocortical carcinoma and capsule invasion were obtained. Patients undergo surgery to remove the tumor, radiotherapy, and chemotherapy.

\section{CONFLICT OF INTEREST}

The author declare there is no conflict of interest of this study.

\section{REFERENCES}

Libe Rossella, 2015. Adrenocortical carcinoma (ACC): diagnosis, prognosis, and treatment. Frontiers in Cell and Developmental Biology, 45 (3): 1-8.

Nieman L \& Ilias I, 2005. Evaluation and treatment of cushing's syndrome. The American Journal of Medicine, 118: 1340-1346.

Nieman L \& Swearingen B, 2013. Cushing's Syndrome and Cushing's Disease. CMM Global : 1-12.

Polat B, Fassnacht M, Pfreundner L, Guckenberger M, 
Bratengeler K, Johanssen S, Kenn W, Hahner S, Allollo

B, \& Flentje M,2009. Radiotherapy in Adrenocortical Carcinoma. American Cancer Society, 115 : 2816-2823.

Price J \& Grossman A, 2007. Differential Diagnosis of Cushing's Syndrome. Arq Bras Endocrinol Metab, 51(8): 11991206.

Raff H, Sharma S, \& Nieman L, 2014. Physiological Basis for the Etiology, Diagnosis, and Treatment of Adrenal Disorders: Cushing's Syndrome, Adrenal Insufficiency, and Congenital Adrenal Hyperplasia. NIH Public Access, 4(2): 739-769.

Rajput R, 2013. Cushing's syndrome - An update in diagnosis and management. JIACM,14 (3-4): 235-241.

Terzolo M, Daffara F, Ardito A, Zaggia B, Basille V, Ferrari L,\& Berruti A, 2013. Management of adrenal cancer: a 2013 update. Journal Endocrinology Investigation : 1-10.

Zini L, Porpiglia F, \& Fassnacht M, 2011. Contemporary Management of Adrenocortical Carcinoma. European Urology, 60: 1055-1065. 